\title{
Clinical Development of New Antibody-Drug Conjugates in Breast Cancer: To Infinity and Beyond
}

\author{
Romualdo Barroso-Sousa ${ }^{1} \cdot$ Sara M. Tolaney ${ }^{2}$ (1) \\ Accepted: 7 February 2021 / Published online: 5 March 2021 \\ (C) The Author(s), under exclusive licence to Springer Nature Switzerland AG part of Springer Nature 2021
}

\begin{abstract}
Metastatic breast cancer remains an incurable disease, and new therapies are needed. One major limitation of chemotherapy is the toxicity associated with higher dose exposure. Antibody-drug conjugates (ADCs) are a complex and evolving class of agents specifically designed with the objective of delivering antineoplastic medicines in the most precise and selectively targeted way. ADCs are composed of four key components: (1) the target antigen, (2) an antibody construct, (3) a payload (most commonly a cytotoxic agent), and (4) a linker moiety that couples the payload and the antibody. In this review, we discuss the clinical development of ADCs for the treatment of breast cancer, focusing on two recently FDA-approved agents, trastuzumab deruxtecan and sacituzumab govitecan, and discuss the ongoing efforts exploring new agents. Finally, we summarize the current portfolio of clinical trials that could change the algorithm of treatment for early and advanced breast cancer.
\end{abstract}

\section{Key Points}

Antibody-drug conjugates (ADCs) are specifically designed with the objective of delivering antineoplastic agents in the most precise and selectively targeted way, increasing the antitumoral efficacy while minimizing toxicity to normal tissues.

ADCs are composed of four key components: the target antigen, an antibody construct, a payload (most commonly a cytotoxic agent), and a linker moiety that couples the payload and the antibody.

The ADCs trastuzumab deruxtecan and sacituzumab govitecan were recently granted FDA approval for the treatment of human epidermal growth factor receptor 2 (HER2)-positive and triple-negative advanced breast cancer, respectively.

Ongoing clinical trials evaluating ADCs are likely to reshape the standard of care for both early and advanced breast cancer.

Sara M. Tolaney

sara_tolaney@dfci.harvard.edu

Oncology Center, Hospital Sírio-Libanês, Brasília, Brazil

2 Breast Oncology Program, Department of Medical Oncology, Dana-Farber Cancer Institute and Harvard Medical School, 450 Brookline Avenue, Yawkey 1257, Boston, MA 02215, USA

\section{Introduction}

Breast cancer is the most frequently diagnosed cancer and the second most common cause of cancer death in women in the USA [1]. In the metastatic setting, despite available therapies, the majority of patients will die from their disease. Thus, new treatments are needed.

Antibody-drug conjugates (ADCs) are designed to deliver antineoplastic medicines precisely and in selectively targeted ways. ADCs are composed of four key components: (1) the target antigen, (2) the antibody construct, (3) a payload (most commonly a cytotoxic agent), and (4) a linker moiety that couples the payload and the antibody [2]. In general, following the binding of the antibody to overexpressed (or specifically expressed) target tumor antigens, the ADC is then internalized and the payload released. The payload release process can be due to proteolytic degradation of the entire ADC molecule, as is the case with trastuzumab emtansine (T-DM1) or due to the cleavage of the linker because of extracellular or intracellular conditions, including low $\mathrm{pH}$ or proteasome-mediated degradation, as in the case of trastuzumab deruxtecan and sacituzumab govitecan (Fig. 1). Advances in the biotechnology associated with the construction of linkers and the emergence of new payloads led to the development of new ADCs. Some ADCs also have activity via bystander effects, leading to off-target cancer cell killing.

In this review, we focus on the clinical development of the recent data that led the US FDA to grant approval for trastuzumab deruxtecan and sacituzumab govitecan in 
breast cancer and on the new agents in development for treating this disease (Table 1).

\section{Antibody-Drug Conjugates (ADCs) Targeting Human Epidermal Growth Factor Receptor 2 (HER2)}

\subsection{Trastuzumab Emtansine}

Trastuzumab emtansine is an ADC that comprises the humanized monoclonal antibody trastuzumab, conjugated via a non-cleavable thioether linker to DM1, a derivative of the naturally occurring maytansinoid toxin. DM1 is a highly potent cytotoxin that inhibits tubulin polymerization and causes death in proliferating cells [3]. T-DM1 has a drugto-antibody ratio (DAR) of 3.5.

T-DM1 was the first ADC to be granted FDA approval. In 2013, based on data from the EMILIA study [4] that demonstrated improved progression-free survival (PFS) and overall survival (OS) in patients treated with T-DM1 compared with patients treated with lapatinib and capecitabine, the drug was approved as a single agent in patients with human epidermal growth factor receptor 2 (HER2)positive metastatic breast cancer (MBC) who have progressed on previous therapy with trastuzumab and a taxane. More recently, the KATHERINE study [5] established T-DM1 as the standard of care in the adjuvant setting for patients with HER2-positive disease treated with neoadjuvant trastuzumab-based therapy who present with residual disease at the time of surgery. The most commonly reported T-DM1-related adverse events are thrombocytopenia and elevated levels of liver enzymes. Patients may also experience neuropathy with prolonged exposure to the drug.

To date, the development of T-DM1 as first-line treatment for HER2-positive MBC or as a neoadjuvant treatment option has not yet been successful. In the phase III MARIANNE trial, T-DM1 with or without pertuzumab showed noninferior PFS to trastuzumab plus a taxane as first-line
Fig. 1 Basic composition of an antibody-drug conjugate (ADC) and its mechanisms of action. (1) ADCs are composed of four key components: the target antigen, an antibody construct, a payload (most commonly a cytotoxic agent), and a linker moiety that will couple the payload and the antibody. Once the ADC is administered (2), there is the binding of the antibody to overexpressed (or specifically expressed) target tumor antigens leading to ADC internalization. Within lysosomes (3), the payload is released when the linker is cleaved by intracellular conditions, such as low $\mathrm{pH}$ or proteasome-mediated degradation. Depending on its mechanism of action (4), the payload will kill tumor cells through DNA damage, such as topoisomerase I inhibitors SN-38 and exatecan, or through microtubule disruption such as emtansine. Additionally, some payloads, such as $\mathrm{SN}-38$ and exatecan, have a membranepermeable nature and can cross cell membranes and exert a cytotoxic effect on bystander tumor cells (5), regardless of target antigen expression levels

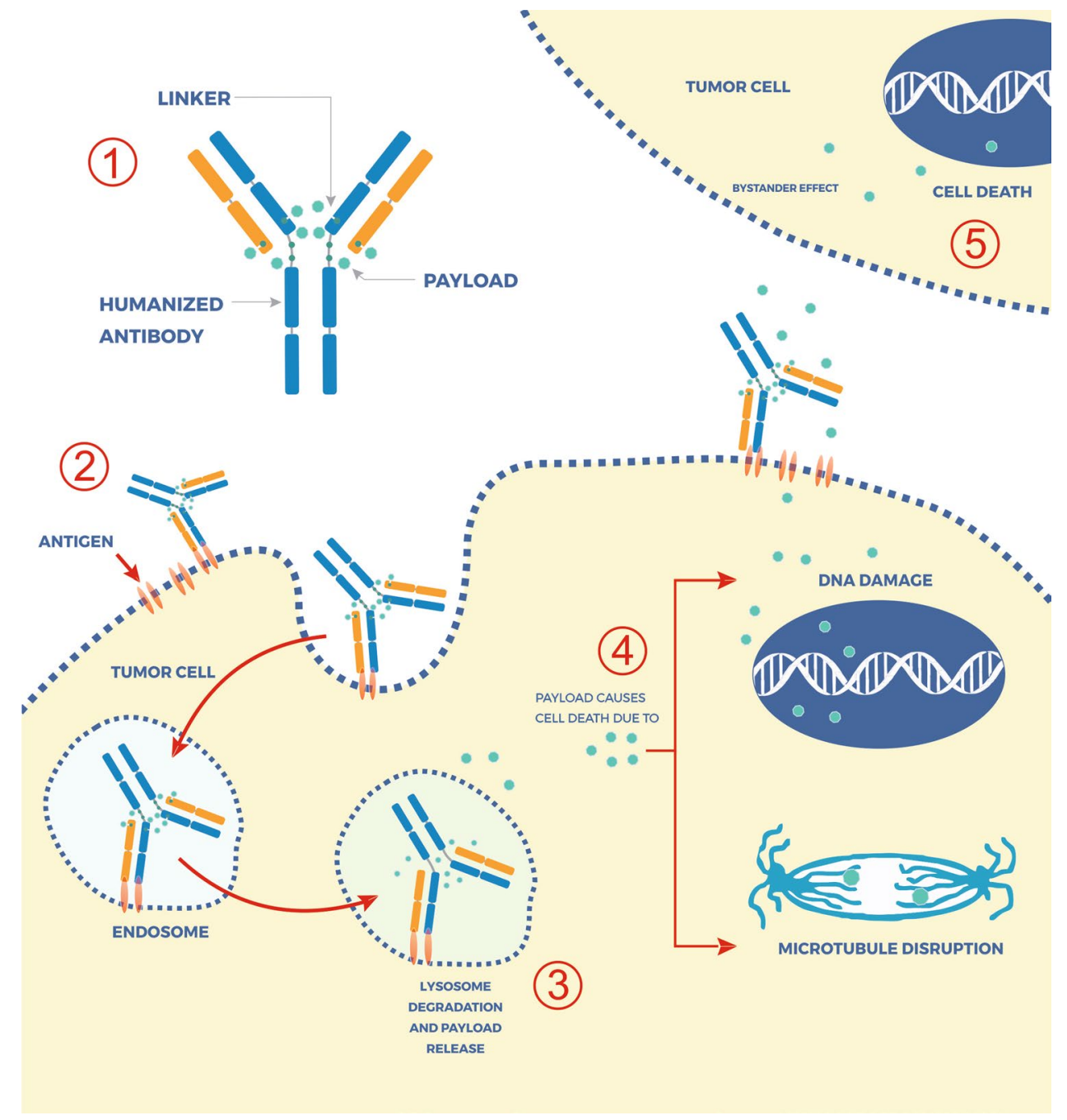


Table 1 Characteristics of the antibody-drug conjugates currently approved or in late stages of development (phase III studies)

\begin{tabular}{|c|c|c|c|c|c|c|}
\hline Agent & Target & $\begin{array}{l}\text { Payload (mechanism of } \\
\text { action) }\end{array}$ & DAR & $\begin{array}{l}\text { Bystander } \\
\text { effect }\end{array}$ & $\begin{array}{l}\text { US FDA-approved indica- } \\
\text { tions }\end{array}$ & Toxicities of special interest \\
\hline $\begin{array}{l}\text { Ado-trastuzumab emtan- } \\
\text { sine }\end{array}$ & HER2 & $\begin{array}{l}\text { Maytansine (antimicrotu- } \\
\text { bule) }\end{array}$ & $3-4$ & No & $\begin{array}{l}\text { HER2+ MBC previously } \\
\text { treated with trastuzumab } \\
\text { and taxane } \\
\text { Early-stage HER2+ BC } \\
\text { with residual disease } \\
\text { following neoadjuvant } \\
\text { therapy (adjuvant) }\end{array}$ & $\begin{array}{l}\text { AST/ALT elevations, } \\
\text { thrombocytopenia, neu- } \\
\text { ropathy }\end{array}$ \\
\hline Trastuzumab deruxtecan & HER2 & $\begin{array}{l}\text { Exatecan (topoisomerase } 1 \\
\text { inhibitor) }\end{array}$ & 8 & Yes & $\begin{array}{l}\text { HER2-positive MBC } \\
\text { previously treated with } \\
\text { trastuzumab, taxane and } \\
\text { T-DM1 }\end{array}$ & $\begin{array}{l}\text { ILD, neutropenia, anemia, } \\
\text { nausea }\end{array}$ \\
\hline $\begin{array}{l}\text { Trastuzumab duocarma- } \\
\text { zine (SYD985) }\end{array}$ & HER2 & Duocarmycin prodrug & 2.8 & Yes & Not approved to date & $\begin{array}{l}\text { Fatigue, conjunctivitis, dry } \\
\text { eyes }\end{array}$ \\
\hline $\begin{array}{l}\text { Disitamab vedotin (RC48- } \\
\text { ADC) }\end{array}$ & HER2 & Monomethyl auristatin E & 4 & No & Not approved to date & $\begin{array}{l}\text { Neutropenia, AST/ALT } \\
\text { elevations }\end{array}$ \\
\hline Sacituzumab govitecan & TROP-2 & $\begin{array}{l}\text { SN-38 (topoisomerase } 1 \\
\text { inhibitor) }\end{array}$ & 7.6 & Yes & $\begin{array}{l}\text { Metastatic TNBC previ- } \\
\text { ously treated with at least } \\
\text { two lines of CT in the } \\
\text { metastatic setting }\end{array}$ & $\begin{array}{l}\text { Neutropenia, anemia, diar- } \\
\text { rhea }\end{array}$ \\
\hline Ladiratuzumab vedotin & LIV-1 & Monomethyl auristatin E & 4 & No & Not approved to date & Neutropenia, anemia \\
\hline
\end{tabular}

$A L T$ alanine transaminase, $A S T$ aspartate aminotransferase, $B C$ breast cancer, $C T$ chemotherapy, DAR drug-to-antibody ratio, HER2+ HER2positive by American Society of Clinical Oncology/College of American Pathologists guidelines, $I L D$ interstitial lung disease, $M B C$ metastatic breast cancer, $T-D M 1$ trastuzumab emtansine, $T N B C$ triple-negative breast cancer

therapy for metastatic HER2-positive disease [6]. However, data from the CLEOPATRA study demonstrated that added pertuzumab to a taxane and trastuzumab improved both PFS and OS [7-9]. Therefore, T-DM1 remained a second-line standard. In the neoadjuvant KRISTINE trial, docetaxel, carboplatin, trastuzumab plus pertuzumab resulted in significantly more patients achieving a pathologic complete response than T-DM1 plus pertuzumab [10], although toxicity was substantially better in the T-DM1 + pertuzumab arm. Importantly, studies combining T-DM1 with the recently approved anti-HER2 tyrosine kinase inhibitor tucatinib are ongoing: the CompassHER2-RD trial (NCT04457596) is evaluating whether the combination of T-DM1 plus tucatinib is superior to T-DM1 alone in the adjuvant setting, and the HER2CLIMB-02 trial (NCT03975647) is evaluating whether the combination of T-DM1 plus tucatinib is superior to T-DM1 alone in patients previously treated with a trastuzumab plus taxane regimen in the metastatic setting.

\subsection{Trastuzumab Deruxtecan}

Trastuzumab deruxtecan (DS-8201 or T-Dxd) is an ADC composed of a humanized anti-HER2 monoclonal antibody with the same amino acid sequence as trastuzumab and a cleavable tetrapeptide-based linker coupled to a potent topoisomerase I inhibitor payload: an exatecan derivative (Table 1). Trastuzumab deruxtecan has a high DAR: eight molecules of the exatecan derivative per monoclonal antibody, which allows the delivery of high concentrations of the payload [11]. Following binding to HER2, the ADC is internalized and trafficked intracellularly to lysosomes [12]. Importantly, while stable in plasma, the linker undergoes selective cleavage by lysosomal cathepsins, which are upregulated in tumor cells [13-16]. Furthermore, given the membrane-permeable nature of the payload, it can cross cell membranes and exert its potent cytotoxic effect on bystander tumor cells regardless of HER2 expression levels [17].

In 2015, a phase I, first-in-human study accrued heavily pretreated patients with advanced HER2-positive breast, or with gastric or gastro-esophageal cancer, with the primary objective of establishing the recommended dose for study expansion and assessing the safety, tolerability, and activity of trastuzumab deruxtecan [11]. Based on preliminary antitumor activity and safety data, the doses of 5.4 and $6.4 \mathrm{mg} /$ $\mathrm{kg}$ were selected as the recommended doses for the expansion phase of this study. In this trial, the maximum tolerated dose was not reached.

\subsubsection{Use in HER2-Positive Breast Cancer}

During the dose-expansion phase of the aforementioned study, the safety, tolerability, and activity of trastuzumab deruxtecan were further assessed in five patient cohorts (Table 2). The study included 115 patients (seven from 
cohort 1; 100 from cohort 2a; and eight from cohort 2e) with HER2-positive breast cancer who received at least one dose of trastuzumab deruxtecan at the recommended doses for expansion of $5.4 \mathrm{mg} / \mathrm{kg}(n=49)$ or $6.4 \mathrm{mg} / \mathrm{kg}(n=66)$ every 3 weeks [18]. Patients in this study had received a median of seven previous cancer therapies (range 5.0-11.0) and 70\% had hormone receptor (HR)-positive tumors. At the time of data cutoff, the median duration of treatment was 8.3 months (interquartile range [IQR] 4.4-12.0), and 60 (52\%) patients had discontinued therapy, most commonly due to progressive disease per RECIST 1.1 in 30 (26\%) patients, clinical progression in $12(10 \%)$, or adverse events in $13(11 \%)$. The most common classes of treatment-emergent adverse events were gastrointestinal and hematological. Notably, there were 20 cases (17\%) of interstitial lung disease (ILD), pneumonitis, or organizing pneumonia, including one grade 3 event and two treatment-related deaths due to pneumonitis. Of the 20 cases, six were observed with $5.4 \mathrm{mg} / \mathrm{kg}$ (6 [12\%] of 49) and 14 with $6.4 \mathrm{mg} / \mathrm{kg}$ doses (14 [21\%] of 66). No cases of decreased ejection fraction were reported. Among the 111 patients included in the efficacy analysis, $66(59.5 \%)$ had a confirmed objective response. The median time to response was 1.6 months, and the median duration of response was 20.7 months. The median PFS was 22.1 months, and the median OS has not been reached. Post hoc analysis showed similar objective response rates (ORRs) independent of previous pertuzumab treatment $(62.5 \%)$, dose group $(5.4 \mathrm{mg} / \mathrm{kg}$ $=56.5 \% ; 6.4 \mathrm{mg} / \mathrm{kg}=61.5 \%$ ), and HR status (HR positive $=59.5 \%$; HR negative $=61.1 \%$ ).

In 2019, the preliminary results of the DESTINYBreast01 study (NCT03248492) were published [19]. This two-part, open-label, single-group, multicenter, phase II study evaluated trastuzumab deruxtecan in patients with HER2-positive MBC who had previously received treatment with trastuzumab emtansine. Of note, patients with untreated or symptomatic brain metastases, a history of noninfectious ILD or pneumonitis resulting in the use of glucocorticoids, or current or suspected ILD or pneumonitis were excluded. In the first part of the study, the investigators explored three different doses of trastuzumab deruxtecan $(5.4,6.4$, and 7.4 $\mathrm{mg} / \mathrm{kg}$ every 3 weeks) to determine a recommended dose; in the second part, the efficacy and safety of the recommended dose was assessed. The study showed a significant relationship between drug exposure and both efficacy outcomes and key adverse events, including ILD. Thus, considering the balance of safety and efficacy, the $5.4 \mathrm{mg} / \mathrm{kg}$ dose was chosen as the recommend dose for the second part of the study. Among the 253 patients who were enrolled and received at least one dose of trastuzumab deruxtecan, 184 received the recommended dose of intravenous trastuzumab deruxtecan of $5.4 \mathrm{mg} / \mathrm{kg}$ every 3 weeks. The median number of previous lines of therapy for metastatic disease was six. After a median follow-up of 11.1 months, the ORR was $60.9 \%$ (112 of 184 patients), with a median duration of response of 14.8 months. The median PFS was 16.4 months. At the time of the data cutoff, $52.1 \%$ who had received the recommended dose had discontinued trastuzumab deruxtecan. The primary reasons for discontinuation were progressive disease according to RECIST 1.1 (28.8\%) and adverse events (15.2\%). During the study, the most common grade 3 or higher adverse events were neutropenia (20.7\%), anemia $(8.7 \%)$, and nausea (7.6\%). Only three patients had a decrease in the left ventricular ejection fraction (two grade 2 and one grade 3 ); those events were asymptomatic, and patients recovered after drug hold. No patients permanently discontinued treatment because of a decrease in the ejection fraction. Drug-related ILD, as determined by an independent adjudication committee, occurred in 25 patients (13.6\%; grade 1 or $2,10.9 \%$; grade 3 or $4,0.5 \%$; and grade $5,2.2 \%$ ).

The robust efficacy data from this single-arm phase II study led the FDA to grant accelerated approval to trastuzumab deruxtecan for patients with unresectable or metastatic HER2-positive breast cancer who have received two or more prior anti-HER2-based regimens in the metastatic setting [20]. In an updated analysis of the DESTINY-Breast01 study, the median PFS was 19.4 months and the estimated 12- and 18-month OS rates were 85\% (95\% confidence interval [CI] 79-90) and 74\% (95\% CI 67-80), respectively. The updated toxicity data revealed a slightly higher rate of ILD of $15.2 \%$ [21].

Ongoing international randomized phase III studies aim to address whether trastuzumab deruxtecan can improve efficacy outcomes compared with other approved anti-HER2based regimens, such as T-DM1 (NCT03523585) or lapatinib plus capecitabine (NCT03529110), in both early and advanced stages of HER2-positive breast cancer. DESTINYBreast05 (NCT04622319) is a phase III, multicenter, randomized, open-label study that will evaluate the efficacy (as assessed by invasive disease-free survival) of trastuzumab deruxtecan versus T-DM1 in patients with high-risk HER2positive primary breast cancer who have residual invasive disease in breast or axillary lymph nodes following neoadjuvant therapy and who meet one of the following high-risk criteria: inoperable breast cancer at presentation (prior to neoadjuvant therapy), defined as clinical stages T4, N0-3, M0 or T1-3, N2-3, M0; operable at presentation, defined as clinical stages $\mathrm{T} 1-3, \mathrm{~N} 0-1, \mathrm{M} 0$, with axillary node-positive disease (ypN1-3) following neoadjuvant therapy (Table 2).

\subsubsection{Use in HER2-Low Breast Cancers}

Between 40 and $50 \%$ of patients with breast cancer have tumors with low HER2 expression (defined as immunohistochemistry [IHC] 1 + or IHC 2 + with no HER2 amplification) [22]. This mixed population, including HR-positive breast cancer and triple-negative breast cancer (TNBC), does 


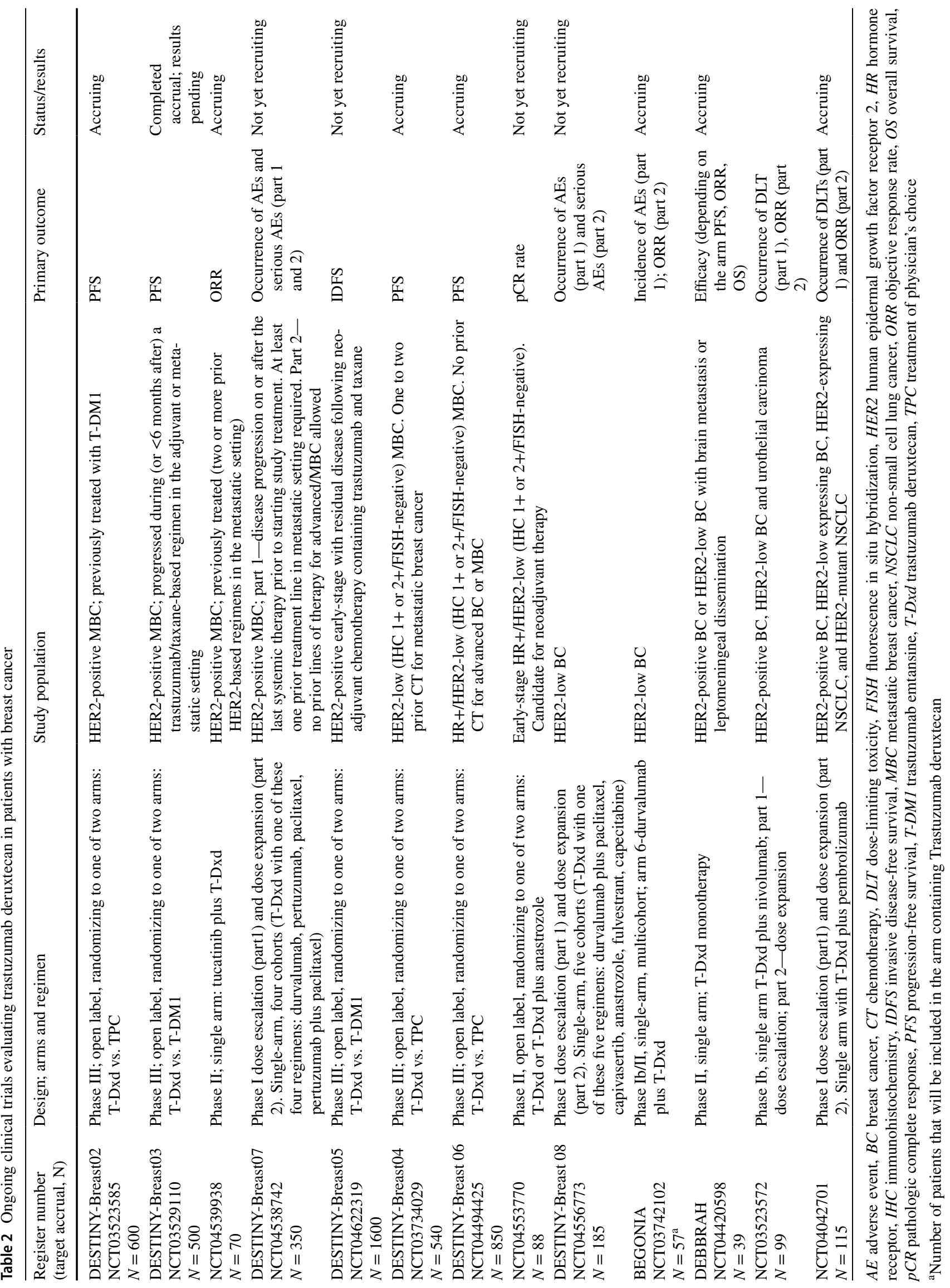


not benefit from the currently approved anti-HER2 therapies. However, in preclinical studies, trastuzumab deruxtecan demonstrated antitumor activity in a variety of tumor types, including those with low HER2 expression [16]. Such activity in HER2-low-expressing tumors may be due to the already mentioned bystander effect in conjunction with the high DAR of trastuzumab deruxtecan with the high potency of payload [16, 17, 23]. Thus, trastuzumab deruxtecan was also tested for the treatment of MBC in patients with low HER2 expression in the previously discussed phase I study (NCT03734029) [24]. During the dose-expansion part of the study, patients with HER2-low breast cancer were enrolled (Table 2). The data of 54 patients with HER2-low advanced/ unresectable or MBC who received at least one dose of trastuzumab deruxtecan at the recommended dose $(5.4 \mathrm{mg} / \mathrm{kg}[n$ $=21]$ or $6.4 \mathrm{mg} / \mathrm{kg}[n=33])$ were jointly reported [24]. All patients had visceral disease at baseline, and most (87.0\%) had HR-positive disease. Although these patients were heavily pretreated (median 7.5 prior therapies), the confirmed ORR by independent central review was $37.0 \%$, the median duration of response was 10.4 months, the median PFS was 11.1 months, and the median OS was 29.4 months. The antitumor activity of trastuzumab deruxtecan was similar between patients with HER $21+(n=28$; ORR $=35.7 \%)$ or $2+(n=26 ;$ ORR $=38.5 \%)$ and patients with $(n=16$; ORR $=43.8 \%)$ or without $(n=38$; ORR $=34.2 \%)$ prior cyclindependent kinase $4 / 6$ inhibitor therapy. Objective responses were less frequent in patients with HR-negative tumors (14.4\%) than in those with HR-positive tumors (40.3\%). Eleven (20.4\%; one with $5.4 \mathrm{mg} / \mathrm{kg}$ and ten with $6.4 \mathrm{mg} / \mathrm{kg}$ ) potential ILD events were reviewed by the independent adjudication committee and eight (14.8\%; all $6.4 \mathrm{mg} / \mathrm{kg}$ ) were attributed to trastuzumab deruxtecan, including three (5.6\%) grade 1, two (3.7\%) grade 2, and three (5.6\%) grade 5 events.

Data from this phase I study suggest a promising antitumor activity of trastuzumab deruxtecan, especially in HRpositive breast cancer, and justify the ongoing randomized, multicenter study phase III studies DESTINY-Breast04 (NCT03734029) and DESTINY-Breast06 (NCT04494425). The primary objective of these studies is to compare the efficacy (PFS) of trastuzumab deruxtecan at the $5.4 \mathrm{mg} / \mathrm{kg}$ dose versus chemotherapy of physician's choice in patients with HER2-low, unresectable, and/or MBC. While DESTINYBreast 04 allows inclusion of any HER2-low breast cancer independently of HR status, DESTINY-Breast06 restricts the inclusion to only HR-positive tumors (Table 2).

\subsubsection{Other Clinical Trials with Trastuzumab Deruxtecan}

Data from the central nervous system (CNS) subgroup included in the DESTINY-Breast01 trial demonstrated a consistent safety and efficacy profile when compared with the overall population [25]. The confirmed ORR for the 24 patients with CNS metastases at baseline was 58.3\%, including $4.2 \%$ with complete response. At median follow-up of 11.0 months, the median PFS in the CNS subgroup was 18.1 versus 16.4 months in the overall population. As per protocol, patients with untreated or progressing brain metastases were not eligible for DESTINY-Breast 01. It is unknown whether trastuzumab deruxtecan is as effective in treating or preventing brain metastasis as has been shown for non-CNS disease. To help address this, a multicohort phase II study is exploring the efficacy of trastuzumab deruxtecan for patients with both HER2-positive or HER2-low breast cancer and untreated or progressive brain metastasis (NCT04420598).

Studies are also exploring trastuzumab deruxtecan in combination with tucatinib and pertuzumab (NCT04539938) and with immune checkpoint inhibitors (NCT03742102; NCT04042701; NCT04556773; NCT04538742). A phase Ib study evaluating trastuzumab deruxtecan in combination with nivolumab that included patients with advanced/MBC (either HER2 positive or HER2 low) (NCT03523572) had 48 patients who received the recommended phase II dose (RP2D) of trastuzumab deruxtecan $(5.4 \mathrm{mg} / \mathrm{kg})$ in combination with nivolumab $360 \mathrm{mg}$ every 3 weeks (HER2 positive, $n=32$; HER2 low, $n=16$ ) [26]. After a median follow-up of approximately 7 months, the confirmed ORR was $59 \%$ in the HER2-positive cohort, with a median PFS of 8.6 months. In the HER2-low cohort, the ORR was 38\% and the median PFS was 6.3 months. Among these 48 patients, five (10.4\%) had adjudicated drug-related ILD, including one fatal case (2.1\%). With similar ORR to trastuzumab deruxtecan monotherapy, longer follow-up and additional studies will be necessary to determine whether this combination provides more clinical benefit than trastuzumab deruxtecan alone. Additionally, other clinical trials are exploring the combination of trastuzumab deruxtecan with more traditional partners, including anastrozole (NCT04553770) and conventional cytotoxic chemotherapy (NCT04556773) (Table 2).

\subsubsection{Management of Trastuzumab Deruxtecan-Associated Interstitial Lung Disease}

Other HER2-directed therapies, including trastuzumab, trastuzumab emtansine, and topoisomerase I inhibitors, have been associated with pulmonary toxicity; however, trastuzumab deruxtecan has higher rates, including some grade 5 events [27].

To evaluate potential risk factors for developing treatment-related ILD, Powell et al. [28] completed a post hoc analysis using pooled data from patients included in two open-label interventional studies with advanced solid tumors $(n=105)(\mathrm{NCT} 02564900)$ and breast cancer $(\mathrm{n}=437)$ (NCT03248492). Patients had received at least one dose of trastuzumab deruxtecan, ranging from 1.6 to $8.0 \mathrm{mg} / \mathrm{kg}$. Compared with the overall study population, patients in the 
breast cancer population developed a higher incidence of any-grade ILD (16.8\% [91/542] in the overall study population vs. $18.1 \%$ [79/437] in the breast cancer population) and developed the disease earlier (median time to onset 208 days in the overall population vs. 134 days in the breast cancer population). The study also showed that patients from Japan were more likely to develop ILD after treatment with trastuzumab deruxtecan than were those of non-Japanese ethnicity. Similarly, a pooled analysis from seven studies evaluating trastuzumab deruxtecan across multiple tumor types found a median time to onset of adjudicated drugrelated ILD cases of 159 (range 46-591) days. In DESTINYBreast01, the median time to onset of lung disease was approximately 6 months (193 days; range 42-535).

Given the limited knowledge about predictors of developing ILD, educating the medical team and patients, and closely monitoring patients for signs and symptoms of ILD (such as fever, cough, or dyspnea), are recommended for early detection. In cases where ILD is suspected, assessment with high-resolution computed tomography, consultation with a pulmonologist, pulmonary function tests, and oxygen saturation should be promptly performed. It is also important to rule out other possible etiologies, including infection, such as with severe acute respiratory syndrome coronavirus 2 (SARS-CoV-2; coronavirus disease 2019 [COVID-19]).

Until more data are available, the recommendation is to hold the next trastuzumab deruxtecan dose in case of grade $1 \mathrm{ILD}$, start patients on systemic steroids $(0.5-1.0 \mathrm{mg} / \mathrm{kg}$ prednisone or equivalent), and resume the drug only if this adverse event is resolved. In addition, for grade 2 or higher ILD, trastuzumab deruxtecan should be permanently discontinued and systemic steroids should be promptly started [29]. Further work is ongoing to evaluate optimal monitoring and management of ILD due to trastuzumab deruxtecan.

\subsection{Trastuzumab Duocarmazine}

Trastuzumab duocarmazine is an ADC composed of a humanized anti-HER 2 monoclonal antibody with the same amino acid sequence as trastuzumab, covalently bound to a linker drug containing duocarmycin, with a DAR of 2.8:1. Following binding to HER2 and endocytosis, the linker is cleaved in the lysosome by proteases that release the active toxin. Duocarmycin alkylates the DNA, resulting in DNA damage in both dividing and non-dividing cells and, ultimately, cell death [30]. Given that proteases such as cathepsin B can be active extracellularly through secretion by malignant cells, there is the potential for a bystander cellkilling effect that is not HER2 mediated [31]. Lastly, trastuzumab duocarmazine showed promising preclinical antitumor activity in solid tumors with varying (low to high) HER2 expression, including breast cancer models [32].
Banerji et al. [33] reported results of a first-in-human, phase I dose-escalation and dose-expansion study (NCT02277717). Patients in this trial had locally advanced or metastatic solid tumors with variable HER2 status and were refractory to standard cancer treatment. A total of 146 patients were enrolled and treated in the dose-expansion phase at the RP2D of $1.2 \mathrm{mg} / \mathrm{kg}$. In this phase, the most common treatment-related adverse events were fatigue (33\%), conjunctivitis $(31 \%)$, and dry eye $(31 \%)$. Grade 3 ocular events were reported in $7 \%$ of patients. There were no treatment-related deaths. A promising ORR of 33\% was reported in patients with HER2-positive breast cancer $(N$ $=48$ ) and, among the 47 patients with HER2-low breast cancer, the response rate was 28 and $40 \%$ for patients with HR-positive and HR-negative breast cancer, respectively.

The ongoing TULIP phase III study is investigating the efficacy (as assessed by PFS) of trastuzumab duocarmazine versus treatment of physician's choice (TPC) (containing an anti-HER2 agent) in patients with HER2-positive MBC who experienced either progression during or after at least two HER2-targeting treatment regimens for locally advanced or metastatic disease or progression during or after (ado-) trastuzumab emtansine treatment (NCT03262935) (Table 3).

\subsection{Disitamab Vedotin}

Disitamab vedotin (RC48-ADC) is an ADC composed of a humanized monoclonal antibody targeting HER2 (disitamab) and a protease cleavable linker covalently coupled to the payload monomethyl auristatin E (MMAE), a synthetic antineoplastic agent (Table 1). Disitamab vedotin has a DAR of 4. Previous studies have shown that disitamab vedotin can kill tumor cells by targeting the HER2 protein on the surface of tumor cells as well as by releasing the payload in lysosomes following endocytosis [34].

In a phase I dose-escalation and dose-expansion study, disitamab vedotin $2.0 \mathrm{mg} / \mathrm{kg}$ every 2 weeks presented with good tolerability and promising efficacy (ORR 46.7\%) in patients with HER2-positive MBC [35]. Grade 3 treatmentrelated adverse events occurred in four patients $(13.3 \%)$, including neutropenia (10\%), leukopenia $(6.7 \%)$, aspartate transaminase elevation (3.3\%), and alanine transaminase elevation (3.3\%). No grade 4 or higher adverse events were observed. Based on these results, a randomized phase II study is evaluating the efficacy of disitamab vedotin 2.0 $\mathrm{mg} / \mathrm{kg}$ administered every 2 weeks versus capecitabine in combination with lapatinib in HER2-positive MBC (NCT03500380). Furthermore, a randomized phase III study will evaluate the efficacy of this ADC versus TPC in patients with low HER2-expressing MBC who experienced progression during or after one line of therapy for $\mathrm{MBC}$ (NCT03262935) (Table 3). 


\section{ADCs Targeting Non-HER2 Proteins}

\subsection{Sacituzumab Govitecan}

Sacituzumab govitecan is an ADC composed of the humanized anti-Trop- 2 monoclonal antibody hRS7 $\operatorname{IgG} 1 \kappa$ and a cleavable CL2A linker coupled to the cytotoxic payload SN-38, an active metabolite of the topoisomerase I inhibitor irinotecan [36] (Table 1). Trop-2 is a calcium signal transducer overexpressed in many epithelial cancers, including breast cancer, and implicated in the promotion of cellular proliferation, survival, and invasion [37-41]. High levels of Trop-2 expression are associated with poor prognosis and worse survival in breast cancer [42, 43]. Sacituzumab govitecan has a high DAR of 7.6 molecules of SN-38 per monoclonal antibody, which allows the delivery of high concentrations of SN-38. Following binding to Trop-2, hRS7 (in free or conjugated form) is internalized and trafficked intracellularly to lysosomes [12]. SN-38 is released throughout antibody degradation followed by hydrolysis of the linker at low $\mathrm{pH}$ that can be found within lysosomes as well as extracellularly in the tumor microenvironment. Thus, given the fact that SN-38 is a membrane-permeable molecule, therapeutic concentration of the drug can also be reached in bystander cells to which the conjugate has not bound [44].

A total of 25 patients with different solid tumors, including four TNBC, participated in the phase I first-in-human study of sacituzumab govitecan and received the ADC at dose levels of $8-18 \mathrm{mg} / \mathrm{kg}$ [36]. The maximum tolerated dose was $12 \mathrm{mg} / \mathrm{kg}$ due to neutropenia, but the doses of 8 and $10 \mathrm{mg} / \mathrm{kg}$ were chosen for further development due to hematologic toxicities in the cycles following the dose of $12 \mathrm{mg} / \mathrm{kg}$.

\subsubsection{Triple-Negative Breast Cancer}

IMMU-132-01 (NCT01631552) was a phase I/II, basket design, open-label, single-group, multicenter trial involving patients with various types of advanced solid cancers who had received at least one previous therapy for metastatic disease [45]. Patients with brain metastasis were excluded if untreated or if they needed to receive high-dose corticosteroids for at least 4 weeks before enrollment. Full data from the cohort with metastatic TNBC was reported in 2019 [46]. Overall, 108 patients received at least one single dose of intravenous sacituzumab govitecan $10 \mathrm{mg} /$ $\mathrm{kg}$ on days 1 and 8 of 21-day cycles until disease progression or unacceptable adverse events. The ORR was $33.3 \%$ according to local assessment (34.3\% according to blinded independent review), including three patients with complete responses $(2.8 \%)$. The median time to response was 2.0 months, and the median duration of response was 7.7 months. The median PFS was 5.5 months, and the median OS was 13.0 months. The most common adverse events were nausea, diarrhea, fatigue, neutropenia, and anemia. Adverse events leading to interruption of treatment occurred in $44 \%$ of patients, and the most common reason was neutropenia. Based on this pivotal study, the FDA granted accelerated approval to sacituzumab govitecan-hziy for the treatment of adult patients with metastatic TNBC who received at least two prior therapies for metastatic disease [47].

The ASCENT study [48] was an international, openlabel, randomized phase III trial that evaluated the efficacy of intravenous sacituzumab govitecan $(10 \mathrm{mg} / \mathrm{kg}$ on days 1 and 8 of a 21-day cycle) versus single-agent TPC (capecitabine, eribulin, vinorelbine, or gemcitabine) in 468 patients with metastatic TNBC who progressed on two or more prior chemotherapies (including a taxane) in the metastatic setting. Of note, patients who progressed within 12 months from the end of (neo)adjuvant therapy were considered as having had a prior line of therapy. The primary endpoint, PFS by central review, was significantly better with sacituzumab govitecan than with TPC (5.6 and 1.7 months, respectively; hazard ratio $0.41 ; p<0.0001$ ). Secondary efficacy endpoints were also significantly improved with sacituzumab govitecan: median OS was 12.1 months with sacituzumab govitecan versus 6.7 months with TPC (hazard ratio $0.48 ; p<0.0001)$, and ORRs were 35 and 5\%, respectively $(p<0.0001)$. Safety data were in concordance with prior studies, and the most common grade 3 or higher adverse events with sacituzumab govitecan were neutropenia $(51 \%)$, diarrhea (10\%), leukopenia (10\%), and febrile neutropenia (6\%). No deaths were related to sacituzumab govitecan. More recently, an exploratory biomarker analysis evaluated the efficacy of sacituzumab govitecan according to Trop-2 expression or germline BRCA1/2 mutation status and showed that the clinical benefit with sacituzumab govitecan versus TPC in the ASCENT study is irrespective of the level of Trop-2 expression or of germline BRCA1/2 mutational status. Notably, better efficacy outcomes (ORR, PFS, and OS) were among sacituzumab govitecan-treated patients with Trop-2 high and Trop-2 median expression subgroups compared with TPC [49]. Furthermore, an exploratory analysis from the ASCENT study specifically in the population with stable brain metastases showed a trend in PFS favoring sacituzumab over TPC (2.8 vs. 1.6 months) [50]. The SWOG S2007 (NCT04647916) phase II study will prospectively evaluate the efficacy of sacituzumab govitecan, as assessed by intracranial ORR, in patients with HER2-negative breast cancer and brain metastases.

In addition, Brenner et al. [51] explored the pharmacokinetic profiles of sacituzumab in patients with breast cancer brain metastases or primary brain tumors who would undergo brain surgery. Before undergoing craniotomy or biopsy, sacituzumab was administered $16 \mathrm{~h}$ preoperatively, 


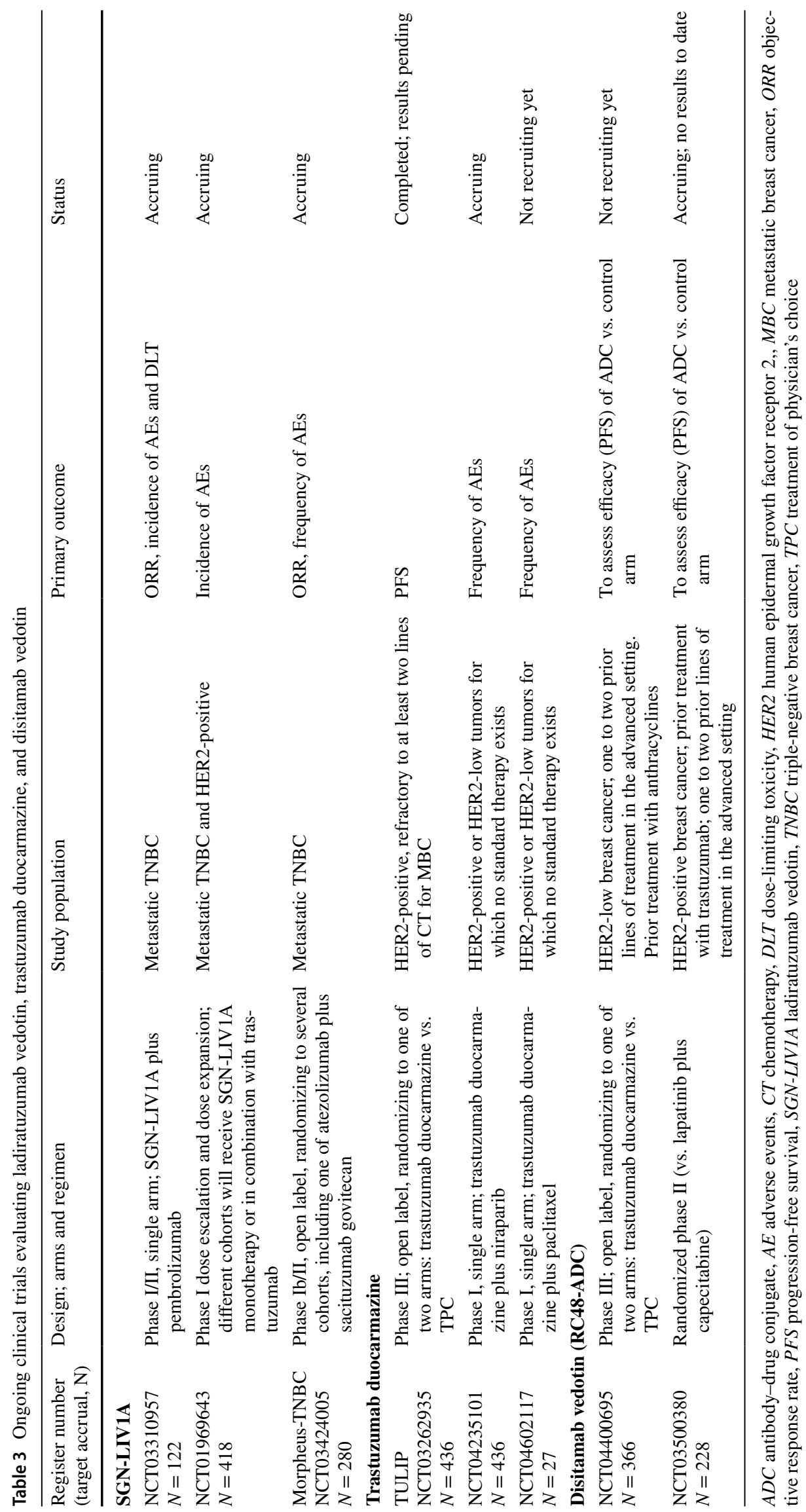


and the pharmacokinetic profile of SN-38 and its derivatives in intracranial tumor tissue, cerebrospinal fluid, and serum were determined. For patients with breast cancer brain metastases, the use of preoperative sacituzumab resulted in therapeutically relevant concentrations of SN-38 at 150-fold mean half maximal inhibitory concentration (IC50) at $18 \mathrm{~h}$ post treatment.

\subsubsection{Use in Hormone Receptor-Positive Breast Cancer}

As part of the previously discussed IMMU-132-01 phase I/II basket study (NCT01631552), a cohort of 54 patients with HR-positive/HER2-negative MBC were treated with intravenous sacituzumab govitecan $10 \mathrm{mg} / \mathrm{kg}$ on days 1 and 8 every 21 -day cycle. These patients had received a median of three prior lines of endocrine therapies and two (range 0-9) prior lines of chemotherapy for MBC. The most common grade $3 / 4$ adverse events were neutropenia $(50 \%)$, anemia (11\%), and diarrhea (7.4\%). After a median follow-up of 11.5 months, sacituzumab govitecan demonstrated a confirmed ORR of $31 \%$ (all partial responses). Responses were durable, with an estimated median duration of response of 7.4 months, PFS of 6.8 months, and OS of 12 months.

These promising data support the ongoing TROPICS-02 trial (NCT03901339), an open-label, randomized, multicenter, global, phase III study that will assess the efficacy of sacituzumab govitecan versus TPC (vinorelbine, capecitabine, eribulin, or gemcitabine) in patients with HR-positive/ HER2-negative MBC, after disease progression on at least two but no more than four prior lines of chemotherapy in the metastatic setting [52]. The primary endpoints for this trial are PFS and ORR according to RECIST 1.1. Key secondary endpoints include OS, clinical benefit rate, and quality of life (Table 4).

\subsubsection{Other Clinical Trials with Sacituzumab Govitecan}

In the early-stage setting of TNBC, a single-arm phase II study is evaluating the efficacy (assessed by the pathologic complete response rate) of this ADC as monotherapy in the neoadjuvant setting (NCT04230109) and a randomized phase III study evaluating the efficacy of adjuvant sacituzumab govitecan versus TPC for residual disease following neoadjuvant therapy. Additionally, there is great interest in the safety, tolerability, and activity of this agent in combination with other therapies in the metastatic setting for HER2negative breast cancer, including immune checkpoint inhibitors (NCT04448886; NCT04468061; NCT03424005) and poly (ADP-ribose) polymerase (PARP) inhibitors in breast cancer (NCT04039230; NCT03992131) (Table 4).

\subsection{Ladiratuzumab Vedotin}

Ladiratuzumab vedotin (SGN-LIV1A) is an ADC composed of a humanized antibody targeting LIV-1, a proteolytically cleavable linker conjugated with the potent microtubuledisrupting agent (MMAE (Table 1). LIV-1 is a multispan transmembrane protein with putative zinc transporter and metalloproteinase activity frequently expressed in breast cancer (both estrogen receptor-positive breast cancer and TNBC), prostate cancer, and melanoma. Preclinical data demonstrate that SGN-LIV1A binds specifically to the extracellular domain of LIV-1, internalizes after antigen binding, and traffics to the lysosome where the payload is released by proteolysis, and causes disruption of microtubulin and induces apoptosis [53].

\subsubsection{Use in Triple-Negative Breast Cancer}

An ongoing phase I study has been evaluating the safety, tolerability, pharmacokinetics, and antitumor activity of SGN-LIV1A (ladiratuzumab vedotin) in patients with LIV1-positive MBC (NCT01969643) [54]. At completion of the dose-escalation stage in patients with HR-positive/HER2negative and TNBC, there were dose-limiting toxicities in 19 evaluable patients; the maximum tolerated dose was $2.8 \mathrm{mg} / \mathrm{kg}$. Expansion cohorts were then opened to further evaluate the safety and antitumor activity of monotherapy in TNBC at 2.0 and $2.5 \mathrm{mg} / \mathrm{kg}$ dosing every 3 weeks. Among the 44 patients with TNBC in the combined dose-escalation and expansion cohorts, the ORR was $32 \%$ and the median PFS was 11.3 weeks. In the entire cohort, the most common grade 3 and 4 adverse events were neutropenia (25\%) and anemia (15\%). Enrollment is ongoing in the triple-negative monotherapy expansion cohort.

The use of SGN-LIV1A as part of the neoadjuvant treatment of patients with early-stage breast cancer was tested as part of the I-SPY 2 trial (NCT0102379). The ADC was given at $2.5 \mathrm{mg} / \mathrm{kg}$ every 3 weeks for four cycles, followed by doxorubicin/cyclophosphamide (AC) every $2-3$ weeks for four cycles. The study did not show superiority of the arm with SGN-LIV1A over the control with regards to estimated pathologic complete response [55]. Additionally, the combination of SGN-LIV1A and immune checkpoint inhibitors has been investigated and two studies are ongoing: one combining the ADC with pembrolizumab (NCT03310957) and another with atezolizumab (NCT03424005) (Table 3). Preliminary data from a phase Ib/II study evaluating the safety, tolerability, activity and RP2D of ladiratuzumab vedotin and pembrolizumab in first-line patients with metastatic TNBC (mTNBC) (NCT03310957) evaluated 51 patients, including 44 patients who received SGN-LIV1A at the RP2D of 2.5 $\mathrm{mg} / \mathrm{kg}$ [56]. The most common grade 3 or higher adverse events were neutropenia, diarrhea, fatigue, hypokalemia, and 


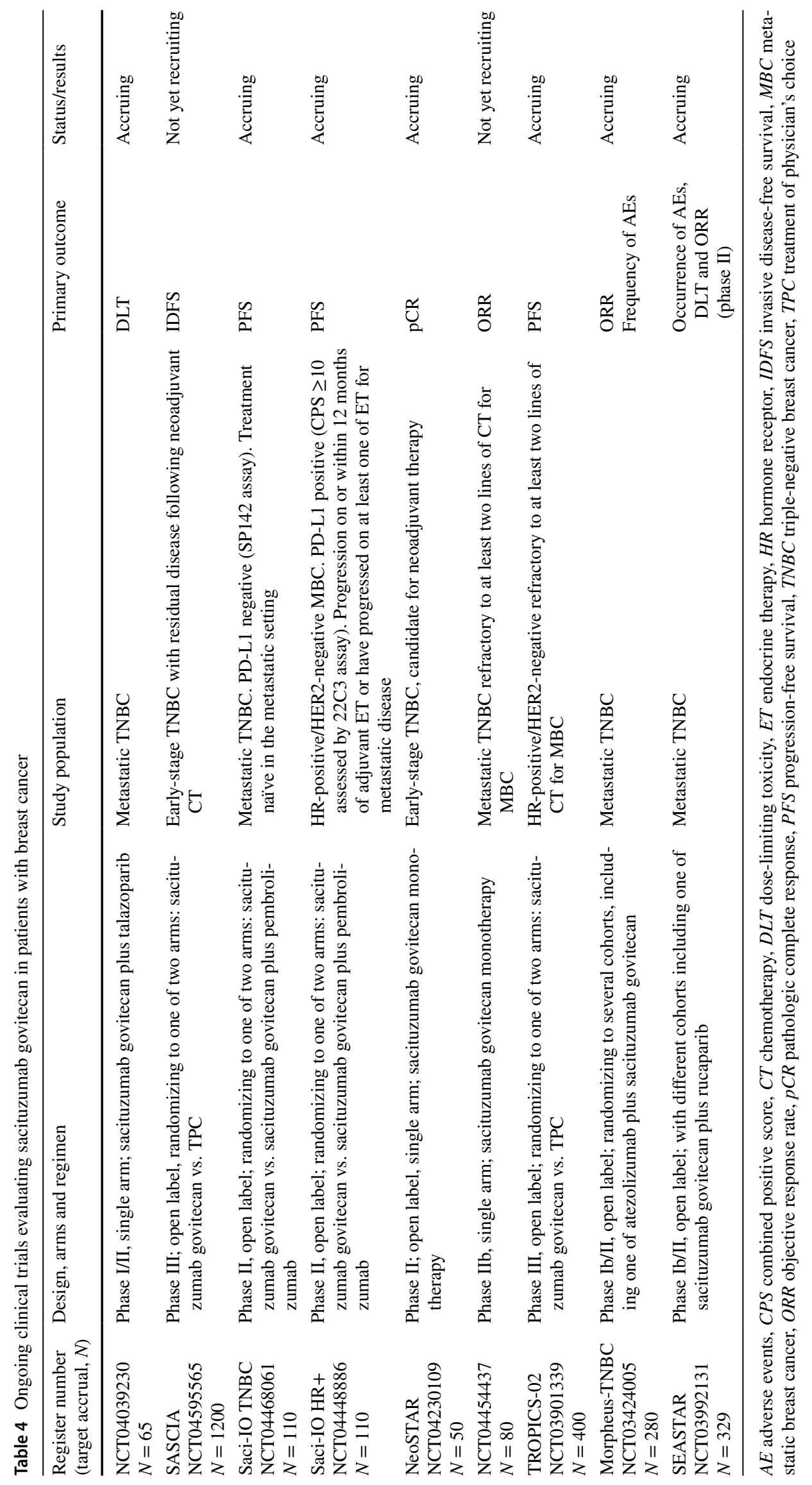




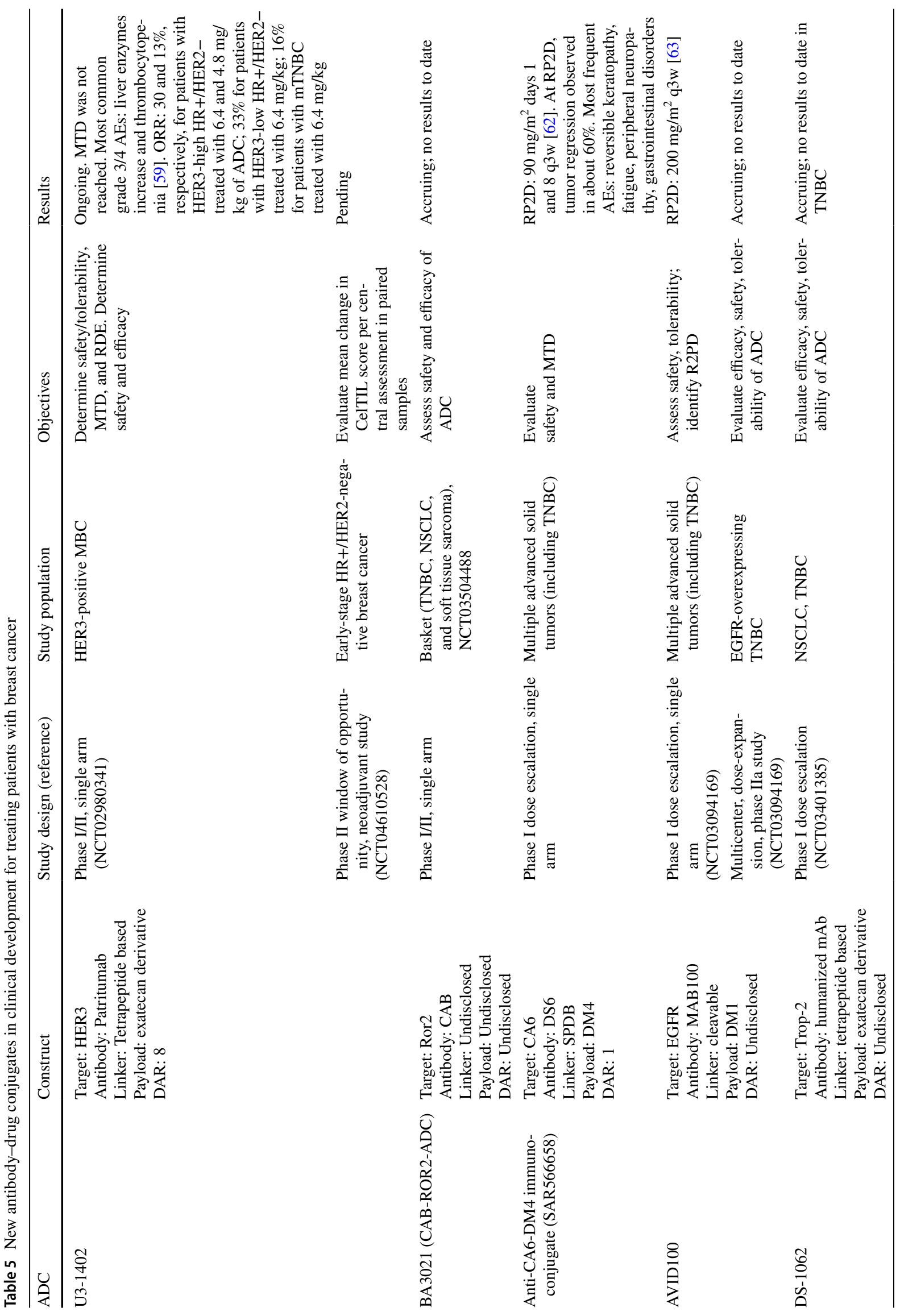




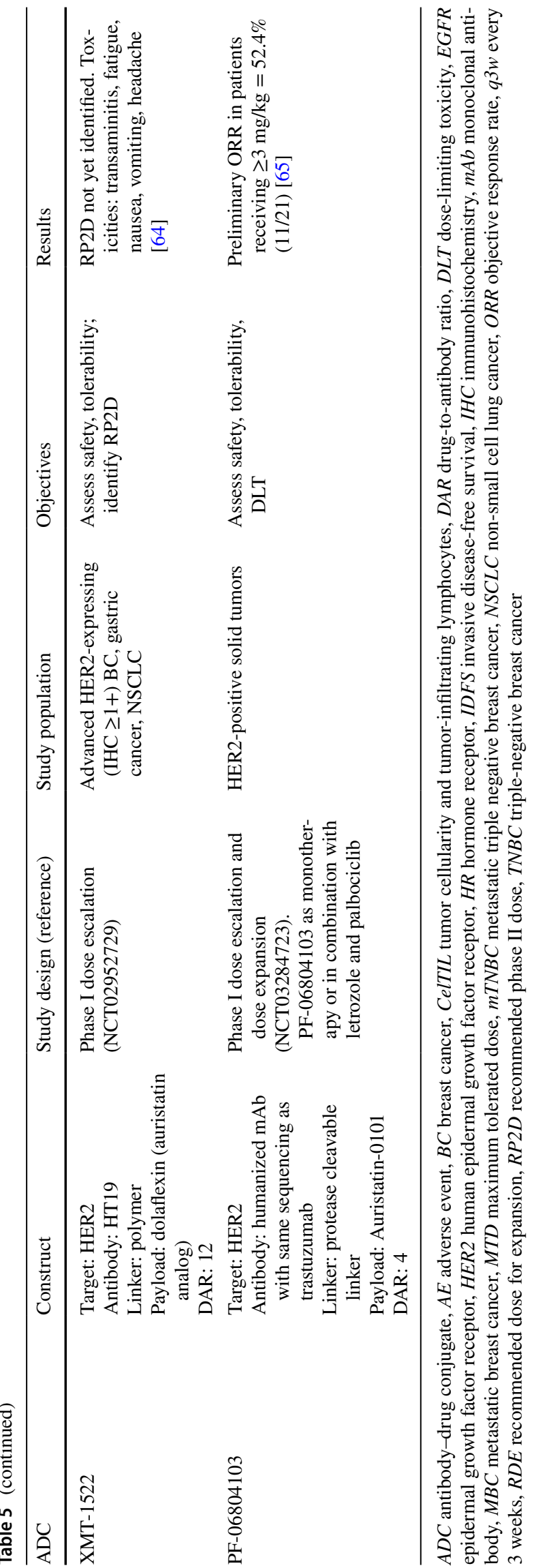

maculo-papular rash (8\% each). The ORR was 54\% among 26 patients who were assessed for efficacy. Overall, the study showed that the combination has a tolerable toxicity profile and promising efficacy in mTNBC (Table 3 ).

\subsection{U3-1402 and Other ADCs}

HER3 is overexpressed in MBC and other tumor types, and overexpression has been associated with poor outcomes $[57,58]$. Patritumab deruxtecan (HER3-DXd; U3-1402) is composed of a fully human anti-HER3 IgG1 monoclonal antibody covalently linked to a topoisomerase I inhibitor payload, an exatecan derivative, via a tetrapeptide-based cleavable linker.

Results from the dose-escalation and dose-finding parts of the NCT02980341/JapicCTI-163401 study demonstrated promising antitumor activity in heavily pretreated patients with HER3-expressing MBC (Table 5) [59, 60]. Recently, data on safety and efficacy for the four separate prespecified dose-expansion cohorts in patients with advanced HRpositive/HER2-negative or mTNBC were presented [61]. Patients with HER3-high/HR-positive/HER2-negative tumors were included in two cohorts to receive HER3-DXd 4.8 or $6.4 \mathrm{mg} / \mathrm{kg}$, whereas patients with HER3-low received $6.4 \mathrm{mg} / \mathrm{kg}$ every 3 weeks. At data cutoff, 85 patients in the HR-positive/HER2-negative cohorts and 31 in the TNBC cohort in the expansion part were evaluable for efficacy. Among 64 patients with HR-positive/HER2-negative HER3high MBC, the ORR was 30 and $13 \%$ for patients treated with 6.4 and $4.8 \mathrm{mg} / \mathrm{kg}$ of ADC, respectively. Additionally, the ORR was 33 and $16 \%$ among 31 patients with HER3low HR-positive/HER2-negative MBC and 31 patients with mTNBC treated with $6.4 \mathrm{mg} / \mathrm{kg}$, respectively. The safety profile of HER3-DXd was manageable, and treatmentrelated adverse events were primarily gastrointestinal or hematologic, and the majority were grade 1 or 2 . The rate of drug-related ILD was $5.2 \%$ (one grade 5).

Several new constructs are currently in the early stages of clinical development, using either HER2 protein as a target or other proteins with potential for treating any subtype of breast cancer. Table 5 summarizes the ADC molecule and clinical data [59, 62-65].

\section{Conclusions}

The emergence of new ADCs with robust efficacy data represents an important therapeutic advance in breast oncology. The advances in the linker-related biochemistry and membrane-permeable nature of both trastuzumab deruxtecan and sacituzumab govitecan certainly helps to explain the success of these agents. Notably, caution is needed to move 
these drugs to the early-stage setting given the potential risk of serious adverse events, including ILD for trastuzumab deruxtecan, and neutropenia and diarrhea for sacituzumab govitecan. Additionally, three other ADCs (ladiratuzumab vedotin, trastuzumab duocarmazine, and disitamab vedotin) are in the final stage of clinical development, and ongoing phase III clinical trials will establish their efficacy versus standard of care. Thus, in the next few years, we might witness a switch from the standard treatment of cytotoxic chemotherapy to anticancer treatment based on ADCs, given either as monotherapy or in combination with other agents.

\section{Declarations}

Funding No external funding was used in the preparation of this manuscript.

Conflict of interest R.B-S. has served as an advisor/consultant to Eli Lilly, Merck Sharp and Dohme, and Roche and has received honoraria from Bard Access, Bristol Myers Squib, Libbs, Eli Lilly, Novartis, Pfizer, Roche, and Zodiac and travel, accommodations, or expenses from Roche and Daiichi-Sankyo. SMT has received institutional research funding from Novartis, Genentech, Eli Lilly, Pfizer, Merck, Exelixis, Eisai, Bristol Meyers Squibb, AstraZeneca, Cyclacel, Immunomedics, Odonate, Sanofi, and Nektar and has served as an advisor/ consultant to Novartis, Eli Lilly, Pfizer, Merck, AstraZeneca, Eisai, Puma, Genentech, Immunomedics, Nektar, Paxman, Athenex, OncoPep, Daiichi-Sankyo, G1 Therapeutics, Gilead, Silverback Therapeutics, Kyowa Kirin Pharmaceuticals, AbbVie, Sanofi, Seattle Genetics, Celldex, Bristol Myers Squibb, and NanoString.

Ethics approval Not applicable.

Consent to participate Not applicable.

Consent for publication Not applicable.

Availability of data and material Not applicable.

Code availability Not applicable.

\section{References}

1. Siegel RL, Miller KD, Jemal A. Cancer statistics, 2016. CA Cancer J Clin. 2016;66(1):7-30.

2. Tolcher AW. The evolution of antibody-drug conjugates: a positive inflexion point. Am Soc Clin Oncol Educ Book. 2020;40:1-8.

3. Hunter FW, Barker HR, Lipert B, et al. Mechanisms of resistance to trastuzumab emtansine (T-DM1) in HER2-positive breast cancer. Br J Cancer. 2020;122(5):603-12.

4. Verma S, Miles D, Gianni L, et al. Trastuzumab emtansine for HER2-positive advanced breast cancer. N Eng1 J Med. 2012;367(19):1783-91.

5. von Minckwitz G, Huang CS, Mano MS, et al. Trastuzumab emtansine for residual invasive HER2-positive breast cancer. N Engl J Med. 2019;380(7):617-28.

6. Perez EA, Barrios C, Eiermann W, et al. Trastuzumab emtansine with or without pertuzumab versus trastuzumab plus taxane for human epidermal growth factor receptor 2-positive, advanced breast cancer: primary results from the phase III MARIANNE study. J Clin Oncol. 2017;35(2):141-8.

7. Baselga J, Cortes J, Kim SB, et al. Pertuzumab plus trastuzumab plus docetaxel for metastatic breast cancer. N Engl J Med. 2012;366(2):109-19.

8. Swain SM, Baselga J, Kim SB, et al. Pertuzumab, trastuzumab, and docetaxel in HER2-positive metastatic breast cancer. N Engl J Med. 2015;372(8):724-34.

9. Swain SM, Miles D, Kim SB, et al. Pertuzumab, trastuzumab, and docetaxel for HER2-positive metastatic breast cancer (CLEOPATRA): end-of-study results from a double-blind, randomised, placebo-controlled, phase 3 study. Lancet Oncol. 2020;21(4):519-30.

10. Hurvitz SA, Martin M, Symmans WF, et al. Neoadjuvant trastuzumab, pertuzumab, and chemotherapy versus trastuzumab emtansine plus pertuzumab in patients with HER2-positive breast cancer (KRISTINE): a randomised, open-label, multicentre, phase 3 trial. Lancet Oncol. 2018;19(1):115-26.

11. Doi T, Shitara K, Naito Y, et al. Safety, pharmacokinetics, and antitumour activity of trastuzumab deruxtecan (DS-8201), a HER2-targeting antibody-drug conjugate, in patients with advanced breast and gastric or gastro-oesophageal tumours: a phase 1 dose-escalation study. Lancet Oncol. 2017;18(11):1512-22.

12. Cardillo TM, Govindan SV, Sharkey RM, et al. Humanized antiTrop-2 IgG-SN-38 conjugate for effective treatment of diverse epithelial cancers: preclinical studies in human cancer xenograft models and monkeys. Clin Cancer Res. 2011;17(10):3157-69.

13. Aggarwal N, Sloane BF. Cathepsin B: multiple roles in cancer. Proteom Clin Appl. 2014;8(5-6):427-37.

14. Ruan J, Zheng H, Fu W, et al. Increased expression of cathepsin L: a novel independent prognostic marker of worse outcome in hepatocellular carcinoma patients. PLoS ONE. 2014;9(11):e112136.

15. Nakada T, Sugihara K, Jikoh T, et al. The latest research and development into the antibody-drug conjugate, [fam-] trastuzumab deruxtecan (DS-8201a), for HER2 cancer therapy. Chem Pharm Bull (Tokyo). 2019;67(3):173-85.

16. Ogitani Y, Aida T, Hagihara K, et al. DS-8201a, a novel HER2targeting ADC with a novel DNA topoisomerase I inhibitor, demonstrates a promising antitumor efficacy with differentiation from T-DM1. Clin Cancer Res. 2016;22(20):5097-108.

17. Ogitani Y, Hagihara K, Oitate M, et al. Bystander killing effect of DS-8201a, a novel anti-human epidermal growth factor receptor 2 antibody-drug conjugate, in tumors with human epidermal growth factor receptor 2 heterogeneity. Cancer Sci. 2016;107(7):1039-46.

18. Tamura K, Tsurutani J, Takahashi S, et al. Trastuzumab deruxtecan (DS-8201a) in patients with advanced HER2-positive breast cancer previously treated with trastuzumab emtansine: a doseexpansion, phase 1 study. Lancet Oncol. 2019;20(6):816-26.

19. Modi S, Saura C, Yamashita T, et al. Trastuzumab deruxtecan in previously treated HER2-positive breast cancer. N Engl J Med. 2020;382(7):610-21.

20. US Food and Drug Administration. FDA approves fam-trastuzumab deruxtecan-nxki for unresectable or metastatic HER2positive breast cancer. https://www.fda.gov/drugs/resources-infor mation-approved-drugs/fda-approves-fam-trastuzumab-derux tecan-nxki-unresectable-or-metastatic-her2-positive-breast-cance r. Accessed 15 Oct 2020.

21. Modi S, Saura C, Yamashita T, et al. Updated results from DESTINY-breast01, a phase 2 trial of trastuzumab deruxtecan (T-DXd ) in HER2 positive metastatic breast cancer [abstract]. Presented at: 2020 San Antonio Breast Cancer Symposium; December 8-11; 2020; Virtual. Poster PD3-06. https://bit.ly/3m7rDoV. Accessed 11 Jan 2021.

22. Tarantino P, Hamilton E, Tolaney SM, et al. HER2-low breast cancer: pathological and clinical landscape. J Clin Oncol. 2020;38(17):1951-62. 
23. Nakada T, Masuda T, Naito H, et al. Novel antibody drug conjugates containing exatecan derivative-based cytotoxic payloads. Bioorg Med Chem Lett. 2016;26(6):1542-5.

24. Modi S, Park H, Murthy RK, et al. Antitumor activity and safety of Trastuzumab Deruxtecan in patients with HER2-low-expressing advanced breast cancer: results from a phase Ib study. J Clin Oncol. 2020;38(17):1887-96.

25. Jerusalem G, Park YH, Yamashita T, et al. CNS metastases in HER2-positive metastatic breast cancer treated with trastuzumab deruxtecan: DESTINY-Breast01 subgroup analyses [abstract]. Ann Oncol. 2020;31(Suppl 2):S63-4.

26. Hamilton E, Shapiro CL, Petrylak D, et al. Trastuzumab deruxtecan (T-DXd; DS-8201) with nivolumab in patients with HER2-expressing, advanced breast cancer: A 2-part, phase 1b, multicenter, open-label study [abstract]. Presented at the 2020 San Antonio Breast Cancer Symposium. https://www.abstractso nline.com/pp8/\#!/9223/presentation/798. Accessed 11 Jan 2021.

27. Hackshaw MD, Danysh HE, Singh J, et al. Incidence of pneumonitis/interstitial lung disease induced by HER2-targeting therapy for HER2-positive metastatic breast cancer. Breast Cancer Res Treat. 2020;183(1):23-39.

28. Powell CA, Camidge DR, Modi S, et al. Risk factors for interstitial lung disease in patients treated with trastuzumab deruxtecan from two interventional studies [abstract]. Ann Oncol. 2020;31(Suppl 4):S357-8.

29. HIGHLIGHTS OF PRESCRIBING INFORMATION (ENHERTUß). https://www.accessdata.fda.gov/drugsatfda_docs/ label/2019/761139s000lbl.pdf. Accessed 11 Jan 2021.

30. Rozhin J, Sameni M, Ziegler G, et al. Pericellular pH affects distribution and secretion of cathepsin B in malignant cells. Cancer Res. 1994;54(24):6517-25.

31. Eaton JS, Miller PE, Mannis MJ, et al. Ocular adverse events associated with antibody-drug conjugates in human clinical trials. J Ocul Pharmacol Ther. 2015;31(10):589-604.

32. van der Lee MM, Groothuis PG, Ubink R, et al. The preclinical profile of the Duocarmycin-based HER2-targeting ADC SYD985 predicts for clinical benefit in low HER2-expressing breast cancers. Mol Cancer Ther. 2015;14(3):692-703.

33. Banerji U, van Herpen CML, Saura C, et al. Trastuzumab duocarmazine in locally advanced and metastatic solid tumours and HER2-expressing breast cancer: a phase 1 dose-escalation and dose-expansion study. Lancet Oncol. 2019;20(8):1124-35.

34. Yao X, Jiang J, Wang X, et al. A novel humanized anti-HER2 antibody conjugated with MMAE exerts potent anti-tumor activity. Breast Cancer Res Treat. 2015;153(1):123-33.

35. Xu B, Wang J, Fang J, et al. Early clinical development of RC48ADC in patients with HER2 positive metastatic breast cancer [abstract]. In: Proceedings of the 2019 San Antonio Breast Cancer Symposium; 2019 Dec 10-14; San Antonio, TX. Philadelphia (PA): AACR; Cancer Res 2020;80(4 Suppl):Abstract nr PD4-06.

36. Starodub AN, Ocean AJ, Shah MA, et al. First-in-human trial of a novel anti-trop- 2 antibody-SN-38 conjugate, sacituzumab govitecan, for the treatment of diverse metastatic solid tumors. Clin Cancer Res. 2015;21(17):3870-8.

37. Ripani E, Sacchetti A, Corda D, et al. Human Trop-2 is a tumor-associated calcium signal transducer. Int $\mathrm{J}$ Cancer. 1998;76(5):671-6.

38. Trerotola M, Cantanelli P, Guerra E, et al. Upregulation of Trop-2 quantitatively stimulates human cancer growth. Oncogene. 2013;32(2):222-33.

39. Shvartsur A, Bonavida B. Trop2 and its overexpression in cancers: regulation and clinical/therapeutic implications. Genes Cancer. 2015;6(3-4):84-105.

40. Basu A, Goldenberg DM, Stein R. The epithelial/carcinoma antigen EGP-1, recognized by monoclonal antibody RS7-3G11, is phosphorylated on serine 303. Int J Cancer. 1995;62(4):472-9.
41. Goldenberg DM, Stein R, Sharkey RM. The emergence of trophoblast cell-surface antigen 2 (TROP-2) as a novel cancer target. Oncotarget. 2018;9(48):28989-9006.

42. Ambrogi F, Fornili M, Boracchi $\mathrm{P}$, et al. Trop-2 is a determinant of breast cancer survival. PLoS ONE. 2014;9(5):e96993.

43. Vidula N, Yau C, Rugo HS. Trop2 gene expression (Trop2e) in primary breast cancer (BC): correlations with clinical and tumor characteristics [abstract]. J Clin Oncol. 2017;35(15_suppl):1075.

44. Sharkey RM, McBride WJ, Cardillo TM, et al. Enhanced delivery of SN-38 to human tumor xenografts with an anti-trop-2-SN-38 antibody conjugate (Sacituzumab Govitecan). Clin Cancer Res. 2015;21(22):5131-8.

45. Bardia A, Mayer IA, Diamond JR, et al. Efficacy and safety of anti-trop-2 antibody drug conjugate Sacituzumab Govitecan (IMMU-132) in heavily pretreated patients with metastatic triplenegative breast cancer. J Clin Oncol. 2017;35(19):2141-8.

46. Bardia A, Mayer IA, Vahdat LT, et al. Sacituzumab Govitecanhziy in refractory metastatic triple-negative breast cancer. N Engl J Med. 2019;380(8):741-51.

47. US Food and Drug Administration. FDA grants accelerated approval to sacituzumab govitecan-hziy for metastatic triple negative breast cancer. https://www.fda.gov/drugs/drug-approvals-anddatabases/fda-grants-accelerated-approval-sacituzumab-govit ecan-hziy-metastatic-triple-negative-breast-cancer. Accessed 15 Oct 2020.

48. Bardia A, Tolaney SM, Loirat D, et al. ASCENT: a randomized phase III study of sacituzumab govitecan (SG) vs treatment of physician's choice (TPC) in patients (pts) with previously treated metastatic triple-negative breast cancer (mTNBC) [abstract]. Ann Oncol. 2020;31(Suppl 4):S1142-215.

49. Hurvitz SA, Tolaney SM, Punie K, et al. Biomarker evaluation in the phase 3 ASCENT study of sacituzumab govitecan versus chemotherapy in patients with metastatic triple-negative breast cancer [abstract]. Presented at: 2020 San Antonio Breast Cancer Symposium; December 8-11, 2020; Virtual. Abstract GS3-06.

50. Diéras V, Weaver R, Tolaney SM, et al. Subgroup analysis of patients with brain metastases from the phase 3 ASCENT study of sacituzumab govitecan versus chemotherapy in metastatic triple-negative breast cancer [abstract]. Presented at the 2020 San Antonio Breast Cancer Symposium. https://www.abstractsonline .com/pp8/\#!/9223/presentation/758. Accessed 11 Jan 2021.

51. Brenner AJ, Pandey R, Chiou J, et al. Delivery and activity of SN-38 by sacituzumab govitecan in breast cancer brain metastases [abstract]. Presented at the 2020 San Antonio Breast Cancer Symposium. https://www.abstractsonline.com/pp8/\#!/9223/prese ntation/756.

52. Rugo HS, Bardia A, Tolaney SM, et al. TROPiCS-02: a phase III study investigating sacituzumab govitecan in the treatment of HR+/HER2 - metastatic breast cancer. Future Oncol (London, England). 2020;16(12):705-15.

53. Sussman D, Smith LM, Anderson ME, et al. SGN-LIV1A: a novel antibody-drug conjugate targeting LIV-1 for the treatment of metastatic breast cancer. Mol Cancer Ther. 2014;13(12):2991-3000.

54. Modi S, Pusztai L, Forero A, et al. Phase 1 study of the antibodydrug conjugate SGN-LIV1A in patients with heavily pretreated triple-negative metastatic breast cancer [abstract]. In: Proceedings of the 2017 San Antonio Breast Cancer Symposium; 2017 Dec 5-9; San Antonio, TX. Philadelphia (PA): AACR; Cancer Res 2018;78(4 Suppl):Abstract nr PD3-14.

55. Beckwith H, Schwab R, Yau C, et al. Evaluation of SGN-LIV1a followed by AC in high-risk HER2 negative stage II/III breast cancer: Results from the I-SPY 2 TRIAL [abstract]. Presented at the 2020 San Antonio Breast Cancer Symposium. https://www. abstractsonline.com/pp8/\#!/9223/presentation/718. Accessed 11 Jan 2021. 
56. Ozaki Y, Mukohara T, Tsurutani J, et al. A multicenter phase II study evaluating the efficacy of nivolumab plus paclitaxel plus bevacizumab triple-combination therapy as a first-line treatment in patients with HER2-negative metastatic breast cancer: WJOG9917B NEWBEAT trial [abstract]. In: Proceedings of the 2019 San Antonio Breast Cancer Symposium; 2019 Dec 10-14; San Antonio, TX. Philadelphia (PA): AACR; Cancer Res 2020;80(4 Suppl):Abstract nr PD1-03.

57. Gala K, Chandarlapaty S. Molecular pathways: HER3 targeted therapy. Clin Cancer Res. 2014;20(6):1410-6.

58. Mota JM, Collier KA, Barros Costa RL, et al. A comprehensive review of heregulins, HER3, and HER4 as potential therapeutic targets in cancer. Oncotarget. 2017;8(51):89284-306.

59. Yonemori K, Masuda N, Takahashi S, et al. Single agent activity of U3-1402, a HER3-targeting antibody-drug conjugate, in HER3-overexpressing metastatic breast cancer: updated results from a phase I/II trial [abstract]. Ann Oncol. 2019;30(Suppl 3):III48.

60. Masuda N, Yonemori K, Takahashi S, et al. Single agent activity of U3-1402, a HER3-targeting antibody-drug conjugate, in HER3-overexpressing metastatic breast cancer: Updated results of a phase 1/2 trial [abstract]. In: Proceedings of the 2018 San Antonio Breast Cancer Symposium; 2018 Dec 4-8; San Antonio, TX. Philadelphia (PA): AACR; Cancer Res 2019;79(4 Suppl):Abstract nr PD1-03.

61. Krop I, Yonemori K, Takahashi S, et al. Safety and efficacy results from the phase 1/2 study of U3-1402, a human epidermal growth factor receptor 3 (HER3)-directed antibody drug conjugate
(ADC), in patients with HER3-expressing metastatic breast cancer (MBC) [abstract]. Presented at the 2020 San Antonio Breast Cancer Sympsoium. https://www.abstractsonline.com/pp8/\#!/9223/ presentation/717. Accessed 11 Jan 2021.

62. Gomez-Roca CA, Boni V, Moreno V, et al. A phase I study of SAR566658, an anti CA6-antibody drug conjugate (ADC), in patients (Pts) with CA6-positive advanced solid tumors (STs)(NCT01156870) [abstract]. J Clin Oncol. 2016;34(15_suppl):2511.

63. Lakhani N, Chandana S, Tolcher A, et al. A Phase Ia/IIa trial of AVID100, an anti-EGFR antibody-drug conjugate [abstract]. In: Proceedings of the American Association for Cancer Research Annual Meeting 2019; 2019 Mar 29-Apr 3; Atlanta, GA. Philadelphia (PA): AACR; Cancer Res 2019;79(13 Suppl):Abstract nr CT056.

64. Hamilton EP, Barve MA, Bardia A, et al. Phase 1 dose escalation of XMT-1522, a novel HER2-targeting antibody-drug conjugate (ADC), in patients (pts) with HER2-expressing breast, lung and gastric tumors [abstract]. J Clin Oncol. 2018;36(15_suppl):2546.

65. Meric-Bernstam F, Calvo E, Moreno V, et al. A phase I dose escalation study evaluating the safety and tolerability of a novel anti-HER2 antibody-drug conjugate (PF-06804103) in patients with HER2-positive solid tumors [abstract]. J Clin Oncol. 2020;38(15_suppl):1039. 